\title{
Global entangling properties of the coupled kicked tops
}

\author{
Rafał Demkowicz-Dobrzański ${ }^{1}$ and Marek Kuśs ${ }^{1,2}$ \\ ${ }^{1}$ Center for Theoretical Physics, Polish Academy of Sciences \\ Aleja Lotników 32/44, 02-668 Warszawa, Poland \\ ${ }^{2}$ Faculty of Mathematics and Sciences, Cardinal Stefan Wyszyński University, Warszawa, Poland
}

(Dated: October 5, 2018)

\begin{abstract}
We study global entangling properties of the system of coupled kicked tops testing various hypotheses and predictions concerning entanglement in quantum chaotic systems. In order to analyze the averaged initial entanglement production rate and the averaged asymptotic entanglement various ensembles of initial product states are evolved. Two different ensembles with natural probability distribution are considered: product states of independent spin-coherent states and product states of random states. It appears that the choice of either of these ensembles results in significantly different averaged entanglement behavior. We investigate also a relation between the averaged asymptotic entanglement and the mean entanglement of eigenvectors of the evolution operator. Lower bound on the averaged asymptotic entanglement is derived, expressed in terms of the eigenvector entanglement.

PACS numbers: 05.45.Mt, 03.67.Mn
\end{abstract}

\section{INTRODUCTION}

Looking for quantum signatures of the classical transition from regular to chaotic dynamics is the field of quantum chaos [1, 2]. Recently it was suggested [3, 4] that entanglement production in a quantum system can be a good indicator of the regular to chaotic transition in its classical counterpart.

In both of the invoked studies [3, 4] it was observed that the presence of chaos enhances the rate at which an initial product state is getting entangled. In the paper [4] Miller and Sarkar considered coupled quantum kicked tops. Single kicked top is a thoroughly studied model in the quantum chaos literature. Depending on the strength of kicks its classical dynamics is either regular or chaotic. Miller and Sarkar studied a system consisting of two identical kicked tops with an additional weak interaction between them. The strength of kicks was chosen in such a way that the classical phase space of a single top was mixed (consisted both of regular tori and chaotic regions). Two tops were initially in a product state of two spin-coherent states. The reason for this choice is that spin-coherent states have a good classical limit, what gives a chance to relate the classical phase space picture to the quantum description. The spin-coherent state of the first top was chosen to lie in a chaotic region while the spin-coherent state of the second one was varied from a regular to a chaotic region. The system was then evolved and it was observed that the rate of entanglement increase (measured as the von Neumann entropy of the reduced density matrix) was higher when the second top was placed in a chaotic region. More quantitatively, it was shown that for different quantum initial states the rate of entanglement increase was proportional to the sum of two positive Lyapunov exponents calculated for the corresponding classical distribution of initial points. These results supported the claim about close relation between chaotic behavior of classical systems and entan- gling properties of their quantum versions.

Further investigations revealed, however, that there is no such direct relation between chaos and entanglement [5, 6, 7, 8, 9, 10, 11]. In particular it was observed that it is rather a specific time correlation function than the Lyapunov exponent itself that determines the entanglement production rate. Strong chaos is able to destroy the time correlations even on a very short time scale and thus it diminishes initial entanglement production rate.

Apart from studying the entanglement production rate, which is a quantity calculated from a short-time behavior of the evolved state, one can also study asymptotic properties of entanglement, i.e. those appearing in the long-time limit. The question whether the asymptotic value of entanglement is related to chaos was posed in [12]. If the coupling strength between kicked tops is large enough to observe the saturation of entanglement within given time, the asymptotic value of entanglement is higher the greater is the chaos parameter (the kick strength). This observation was made starting with an initial state in form of a product of two spin-coherent states placed in a region which for low kick strengths was regular, contained a part of a separatrix for stronger kicks, and eventually became chaotic for very strong kicks.

In the present paper we also concentrate on the case of coupled kicked tops. In most of the previous studies the attention was focused on analyzing how an initial product state of certain spin-coherent states evolves in time. Here we would like to address a more general problem. Namely, how does the strength of chaos influence the global entangling properties of the evolution of the kicked tops. By global we mean properties not depending on a specific choice of initial product state. In other words this is the question of whether the entangling capabilities of the transformation depend on the strength of chaos. Global entangling properties were analyzed in the kicked Ising spin chain model [8], where evolution of 
random states was investigated, and decrease of entanglement production rate with the increase of chaos was observed.

Another approach often used [13] in determining the entangling power of an operation consists of analyzing its eigenvectors. In the case of periodically driven systems these are the eigenvectors of an unitary operator $U$ corresponding to the one-period evolution. The degree of eigenvector entanglement is then regarded as an information about entangling properties of the evolution. We show that the information about entanglement of eigenvectors does not, however, give the full picture of entanglement properties of the evolution, but only a rough estimate of the asymptotic behavior of entanglement of evolved states (see Sec VIA).

In order to discuss entangling properties of the evolution of the kicked tops, we evolve not a single product state, but a whole ensemble of random product states, chosen uniformly with respect to action of $\mathrm{SU}\left(d_{1}\right) \otimes$ $\mathrm{SU}\left(d_{2}\right)$ group, where $d_{1}, d_{2}$ are the Hilbert space dimensions of the two tops. We calculate the averaged asymptotic entanglement approached by evolved states, and the initial rate of entanglement production. All pure states are treated on equal footing here. Spin coherent states are as good as any other pure state. This way of averaging gives us information about the entangling power of evolution, as defined in [14].

Additionally we shall calculate the entanglement production when initial states are products of two spincoherent states, where each spin-coherent state, parameterized by two spherical angles $\theta, \phi$, is taken independently from the ensemble with uniform probability density on the unit sphere. The two ways of averaging give qualitatively different results. We shall analyze the results with the help of the perturbative approach [7, 10] and the analysis of the entanglement of eigenvectors.

The observation that is worth mentioning here is that even in a very regular regime the entangling power is extremely high - higher than in chaotic cases - both when discussing the asymptotic entanglement behavior and the initial entanglement production rate.

Our results are another step to reveal the relation between chaotic vs. regular motion and entanglement production.

\section{ENTANGLEMENT}

Entanglement is a purely quantum phenomenon, dividing states of a composite quantum systems into two classes: those which can be written as products of some states of the (two in this case) subsystems:

$$
|\Psi\rangle=\left|\psi_{1}\right\rangle \otimes\left|\psi_{2}\right\rangle
$$

called product states, and all other which cannot be written in the form (2.1) but instead involve a genuine, non- trivial linear combination

$$
|\Psi\rangle=\sum_{i j} c_{i j}\left|\psi_{1 i}\right\rangle \otimes\left|\psi_{2 j}\right\rangle .
$$

The definition above applies only when one deals with pure states. The notion of entanglement for mixed states is more subtle [15], and it is very difficult in general to determine whether a given mixed state is entangled or not. In this paper the state of the composite quantum system we consider is always pure.

For a given pure state $|\Psi\rangle$ it is easy to check whether it is entangled or not. Observe that if the state is a product one, averaging over one of the subsystems of the corresponding density matrix $\rho=|\Psi\rangle\langle\Psi|$ gives a pure state of the remaining subsystem:

$$
\rho_{1}:=\operatorname{Tr}_{2}(\rho)=\left|\psi_{1}\right\rangle\left\langle\psi_{1}\right|,
$$

whereas the same procedure applied to an entangled state produces necessarily a genuine mixed state. This observation can be further quantified by calculating the linear entropy for the reduced density matrix

$$
S_{L}:=1-\operatorname{Tr}\left(\rho_{1}^{2}\right),
$$

which vanishes for product states and reaches the maximal value:

$$
S_{L}^{\max }=1-1 / d,
$$

where $d=\min \left(d_{1}, d_{2}\right)$ is the Hilbert space dimension of the smaller subsystem, for the "maximally entangled state" (by definition this is the state which reduces to the "maximally mixed" state of a subsystem - the latter is characterized by a diagonal density matrix with equal entries). In this sense $S$ is a measure of entanglement for pure states of a composite system.

Another often used measure of entanglement for pure states is the von Neumann entropy of the reduced density matrix:

$$
S_{\mathrm{vN}}=-\operatorname{Tr}\left(\rho_{1} \log _{2} \rho_{1}\right) .
$$

For product states $S_{\mathrm{vN}}=0$, as the reduced density matrix $\rho_{1}$ is also pure, while for the maximally entangled states the von Neumann entropy of the reduced density matrix takes the highest value $S_{\mathrm{vN}}^{\max }=\log _{2} d$. Contrary to the linear entropy, the above measure of entanglement has a nice operational meaning in terms of the number of maximally entangled states that can be distilled from a given number of non-maximally entangled states [16].

Actually all quantities which do not increase under local operations (ie. operations acting separately in each subsystem) and classical communication quantify in some way the amount of entanglement present in a state. These in general are called entanglement monotones [17]. Linear and von Neumann entropy discussed above are examples of such entanglement monotones for pure states.

In the following we shall use the linear entropy (2.4) as the measure of entanglement. We choose this measure, 
instead of the von Neumann entropy (2.6), as linear entropy is easier to calculate and there is a perturbative formula for initial growth of linear entropy [7, 10] in weakly coupled systems which we shall use. Furthermore, in the investigations concerning relation between chaos and entanglement, where both von Neumann and linear entropy where calculated [7, 12, 18] no qualitative difference in the behavior of the two was found, thus the choice of either of them is not crucial.

It is argued that the presence of entanglement is important in many novel applications of quantum information processing [19], which explains the prominence enjoyed by this phenomenon in many recent investigations. In our study we shall concentrate only on the interplay between production of entanglement in a composite quantum system and its chaotic properties.

\section{COUPLED KICKED TOPS}

The kicked top is a paradigmatic model for studying quantum chaos in finite-dimensional Hilbert spaces [20]. It is a particle with the total spin $j$ and the dynamics generated by the Hamiltonian

$$
H=p J_{y}+\frac{k}{2 j} J_{z}^{2} \sum_{n=-\infty}^{\infty} \delta(t-n) .
$$

Here $J_{y}$ and $J_{z}$ are the components of the angular momentum operator fulfilling the standard commutation relations $\left[J_{y}, J_{z}\right]=i J_{x}$, etc. The time dependence takes the form of an infinite train of delta-shaped pulses ("kicks") perturbing the free rotation periodically in time. The quantities $p$ and $k$ are adjustable parameters of the model. The latter, called the kick-strength, is scaled by the total spin $j$-observe that the total angular momentum $J^{2}=J_{x}^{2}+J_{y}^{2}+J_{z}^{2}$ is conserved, $\left[J^{2}, H\right]=0$, hence we can investigate the dynamics of the model for each value of $j$ independently, restricting the discussion to the appropriate $[(2 j+1) \times(2 j+1)]$-dimensional space. To exhibit various interesting dynamical aspects of the model it is enough to change one of the parameters. In the following we put $p=\pi / 2$ and vary $k$.

The unitary time evolution operator transporting the wave function of the kicked top in time over one period of the perturbation,

$$
U=\exp \left(-i \frac{k}{2 j} J_{z}^{2}\right) \exp \left(-i \frac{\pi}{2} J_{y}\right)
$$

generates the Heisenberg equations of motion for the angular momentum operators $J_{x}, J_{y}$, and $J_{z}$

$$
\begin{aligned}
J_{x}^{\prime} & =U^{\dagger} J_{x} U=\frac{1}{2}\left(J_{z}+i J_{y}\right) e^{-i(k / j)\left(J_{x}-1 / 2\right)}+\text { h.c., } \\
J_{y}^{\prime} & =U^{\dagger} J_{y} U=\frac{1}{2 i}\left(-J_{z}+i J_{y}\right) e^{-i(k / j)\left(J_{x}-1 / 2\right)}+\text { h.c. } \\
J_{z}^{\prime} & =U^{\dagger} J_{x} U=-J_{x}
\end{aligned}
$$

giving the operators $J_{x}^{\prime}, J_{y}^{\prime}$, and $J_{z}^{\prime}$ at time $t=n+1$ in terms of their predecessors $J_{x}, J_{y}$, and $J_{z}$ at time $t=n$.
As in all studies of quantum chaotic phenomena we are ultimately interested in comparing quantum and classical dynamics of the model. In the present the Planck constant has been set to unity hence the classical limit corresponds to $j \rightarrow \infty$ limit ("large quantum numbers"). More formally one defines the quantities $X, Y$ and $Z$ as averages of $J_{x} / j, J_{y} / j$, and $J_{z} / j$ calculated in the initial state of the system. In comparing classical and quantum behavior it is reasonable to take as an initial state some minimum uncertainty state, in belief that (at least in the large $j$ limit) the evolution of averages will be well represented by the single classical trajectory starting from the point in the phase space around which the initial quantum state of minimal uncertainty is concentrated. Obviously, there is no particular reason to distinguish such states when investigating purely quantum properties like entanglement production. Appropriate minimum uncertainty states for spin $j$ particles (so called angular momentum coherent states) can be generated from the $|j, j\rangle$ state [i.e. the common eigenstate of $J_{z}$ and $J^{2}$ with the eigenvalues $m=j$ and $j(j+1)$ respectively] by unitary rotations

$$
|\theta, \phi\rangle=\left(1+|\gamma|^{2}\right)^{-j} e^{\gamma\left(J_{x}-i J_{y}\right)}|j, j\rangle, \quad \gamma=e^{i \phi} \tan \frac{\theta}{2},
$$

and the above described procedure leads to the following classical mapping:

$$
\begin{aligned}
X^{\prime} & =Z \cos (k X)+Y \sin (k X), \\
Y^{\prime} & =-Z \sin (k X)+Y \cos (k X), \\
Z^{\prime} & =-X
\end{aligned}
$$

A detailed analysis of the classical dynamics (3.5) is given in [20], let us only summarize that the system is integrable for $k=0$ and becomes visibly chaotic when $k>2$. For $k$ around 3 the phase space exhibits well developed mixed structure with few regular islands embedded in the chaotic see. When $k \approx 6$ islands of stability, although present, are very small and the chaos can be treated as fully developed for all practical purposes. From the point of view of quantum chaos, the islands are negligible if their phase-space area is smaller then the effective value of the Planck constant $(1 / j$ in our case), which will be the case in our calculations.

In order to achieve our ultimate goal, ie. the investigation of parallels between chaos and entanglement we follow the idea of Miller and Sarkar [4] and consider two coupled kicked tops with the Hamiltonian

$$
H=H_{1}+H_{2}+H_{I}
$$

where $H_{1}$ and $H_{2}$ are the Hamiltonians of independent kicked tops (3.1) expressed in terms of the operators $J_{x_{1}}, J_{y_{1}}, J_{z_{1}}$ and $J_{x_{2}}, J_{y_{2}}, J_{z_{2}}$ pertaining to each individual top, whereas $H_{I}$ is a nonlinear coupling term

$$
H_{I}=\frac{\epsilon}{j} J_{z_{1}} J_{z_{2}} \sum_{n=-\infty}^{\infty} \delta(t-n)
$$


The procedure of obtaining the classical evolution equations is exactly the same as the one for a single top described above, and yields [12]:

$$
\begin{aligned}
X_{1}^{\prime} & =Z_{1} \cos \Delta_{12}+Y_{1} \sin \Delta_{12} \\
Y_{1}^{\prime} & =-Z_{1} \sin \Delta_{12}+Y_{1} \cos \Delta_{12} \\
Z_{1}^{\prime} & =-X_{1} \\
X_{2}^{\prime} & =Z_{2} \cos \Delta_{21}+Y_{2} \sin \Delta_{21} \\
Y_{2}^{\prime} & =-Z_{2} \sin \Delta_{21}+Y_{2} \cos \Delta_{21} \\
Z_{2}^{\prime} & =-X_{2},
\end{aligned}
$$

where $\Delta_{12}=k X_{1}+\epsilon X_{2}, \Delta_{21}=k X_{2}+\epsilon X_{1}$. In the most of the following the coupling strengths $\epsilon$ will be small in comparison with $k$, it means that the degree of chaos in the system is determined solely by properties of dynamics of individual tops.

\section{ENTANGLING POWER}

The main idea behind quantifying the entangling power of quantum evolution is to measure the ability to produce an entangled state out of an initial product state in the course of the quantum evolution. Although for particular reasons or applications we can choose a concrete initial state and follow evolution of its entanglement properties when time passes, such a history would definitely bear a lot of imprints of the initial state we chose to start with. In our study we are more interested in entanglement capabilities of the system itself, so it is more reasonable to take the average over some set of initial states - the idea advanced by Zanardi [14]

$$
e_{p}(U)=\left\langle\left\langle S\left[U\left(\left|\psi_{1}\right\rangle \otimes\left|\psi_{2}\right\rangle\right)\right]\right\rangle\right\rangle_{\psi_{1}, \psi_{2}} .
$$

In the above formula $S$ is some appropriate measure of entanglement (in our case it will be the linear entropy), and $\langle\langle\cdot\rangle\rangle_{\psi_{1}, \psi_{2}}$ denotes averaging over a set of initial product states $\left|\psi_{1}\right\rangle \otimes\left|\psi_{2}\right\rangle$.

The averaging procedure, however, needs more detailed reflection. As mentioned in the previous section, when investigating the quantum-classical correspondence, it is reasonable to take as an initial state a spin-coherent state and, consequently, a product of two such states for a composite system. The averaging amounts to integrating over the whole set of spincoherent states parametrized by the spherical angles $\theta_{i}$ and $\phi_{i}, i=1,2$ in (3.4). In order not to distinguish any particular initial state, we average over products of two spin-coherent states each parameterized by $\theta_{i}$ and $\phi_{i}$ taken independently from the ensemble with the uniform probability density on the unit sphere. This kind of averaging will be denoted as $\mathrm{SU}(2) \times \mathrm{SU}(2)$ averaging, as our ensemble is invariant under the action of rotation in either of subsystems.

As already written, there is no particular reason for such a choice of the set of initial states when global entangling production properties of our system are investigated. Instead one can average over the whole set of initial product states. In order to treat all pure product states on equal footing one should choose the ensemble of product states with probability distribution invariant under the action of $\mathrm{SU}\left(d_{1}\right) \times \mathrm{SU}\left(d_{2}\right)$, where $d_{1}, d_{2}$ are the Hilbert space dimensions for the subsystems. In this way we obtain a natural ensemble of random product states.

It is not a surprise that $\mathrm{SU}(2) \times \mathrm{SU}(2)$ and $\mathrm{SU}\left(d_{1}\right) \times$ $\mathrm{SU}\left(d_{2}\right)$ averages can lead to different quantitative estimates of the entangling power; what is more important they differ also qualitatively.

One can also think about other characterizations of entangling capabilities of quantum evolution operators. For example, entanglement properties of eigenvectors of $U$ can give some information about possible entanglement production. The matter, however, is rather subtle, as it will be clear from the subsequent discussion.

\section{NUMERICAL RESULTS FOR COUPLED KICKED TOPS}

In this section we present the main results of the numerical calculations of the evolution of the coupled kicked tops. The spins of our two tops are respectively $j_{1}=19.5$ and $j_{2}=20$. This choice is an effect of a compromise. The spins should be high enough to allow classical correspondence and low enough to be numerically tractable. Other authors, who considered higher spins $(j=40, j=80)$, were able to perform their calculations as they were evolving only a few different states. Our calculation are performed on ensembles consisting of several hundreds of states. Consequently we sacrifice the spin magnitude for the sake of being able to perform averaging over many states. The chosen spins $j_{1}, j_{2}$ are not equal. This does not change evolution significantly (as compared with $j_{1}=j_{2}=20$ ), but removes degeneracy among eigenstates of one-period evolution operator $U$. Lack of degeneracy is essential for the eigenvectors entanglement analysis, which will be explained later.

The coupling strength is chosen to be $\epsilon=0.01$, while the strength of kicks $k$ (equal for both tops) will be varied from $k=0$ to $k=6$. Chaos enters the classical dynamics of a single top at $k \approx 2$. The coupling constant is small enough to assure that all chaotic behavior is due to the kicks of the tops and not their interaction.

\section{A. Entanglement evolution}

In Fig. 1 and Fig. 2 we show calculations for 1000 iterations of tops evolution. In Fig. 1 the evolution of linear entropy averaged over 100 random initial spin-coherent product states is presented.

During the first 1000 kicks most of the curves saturate to some asymptotic value. The only exception, $k=0.01$, requires a little more time to saturate. The saturation of entanglement in the evolution of the kicked tops is present also in the case of single spin-coherent product 


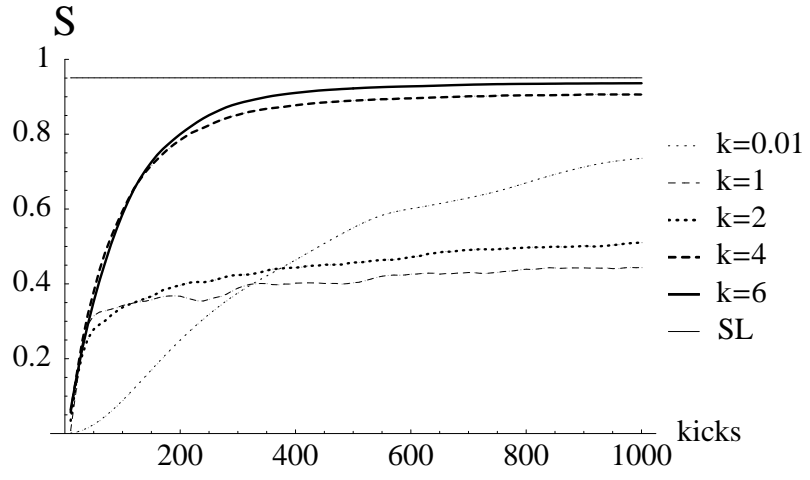

FIG. 1: The time evolution of the linear entropy averaged over initial spin-coherent product states $\left(S_{\mathrm{SU}(2)}\right)$, calculated for different chaoticity parameters $k$ of subsystems (different kick strengths). Spin magnitudes of two interacting tops are taken respectively $j_{1}=19.5, j_{2}=20$ and the coupling strength $\epsilon=0.01$. The evolution time comprises 1000 kicks. The statistical limit (SL) for entanglement is drawn with the solid line and corresponds to the average entanglement of a randomly chosen state of the composite system. Increasing the chaoticity parameter causes in general an increase in the asymptotic value of entanglement (with the exception of the case $k=0.01)$. Initial entanglement growth is extremely slow for very regular dynamics $(k=0.01)$

state evolution - the averaging over spin-coherent states is not necessary, yet the averaged curves have smoother behavior. Different curves saturate to different asymptotic values. This observation was made in [12], where it was pointed out that higher is the chaos parameter $k$, the higher is also the asymptotic value of entanglement reached by the spin-coherent product state $|\theta, \phi\rangle \otimes|\theta, \phi\rangle$ $(\theta=0.89, \phi=0.63)$. Our averaged results confirm this observation only partially. While the asymptotic value indeed increases with $k$ for $k \geq 1$, it is also quite high in the nonchaotic regime $-k=0.01$. For $k=0.01$ the asymptotic value is higher than that for $k=1, k=2$. Opposite to the nonmonotonic behavior of the asymptotic values, the initial entanglement production rates seem to increase monotonically with $k$. We shall discuss these observation more thoroughly in the following. The statistical limit (SL) calculated as an average entanglement of a random pure state of the full system [21] is drawn with a thin solid line.

In Fig. 2 we present the result of averaging over 100 random product states with the probability distribution invariant under the action of the $\mathrm{SU}\left(d_{1}\right) \times \mathrm{SU}\left(d_{2}\right)$ group, where $d_{1}=2 j_{1}+1, d_{2}=2 j_{2}+1$ are the dimensions of subsystems (the state of one top is chosen independently from the state of the other). We generated random product states using random unitary matrices, distributed uniformly according to the $\mathrm{SU}\left(d_{1}\right) \times \mathrm{SU}\left(d_{2}\right)$ Haar measure, applied to a fixed product state [22]. Different asymptotic values of entanglement for different $k$ is again visible. A monotonic increase of the asymptotic

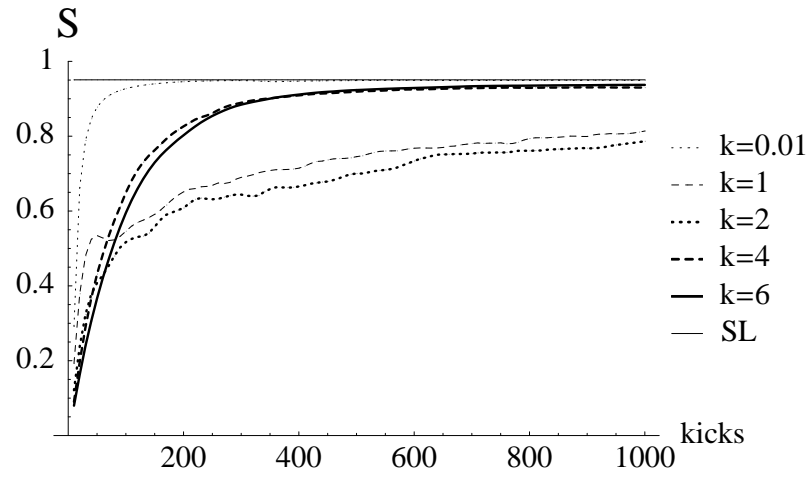

FIG. 2: The time evolution of the linear entropy averaged over the ensemble of initial random product states $\left(S_{\mathrm{SU}(d)}\right)$, with the probability distribution invariant under the action of the $\mathrm{SU}\left(d_{1}\right) \times \mathrm{SU}\left(d_{2}\right)$ group, where $d_{1}=2 j_{1}+1, d_{2}=2 j_{2}+1$ are the dimensions of the subsystems. Different curves correspond to different chaoticity parameters $k$ of the subsystems (different kick strengths). Spin magnitudes of two interacting tops are taken respectively $j_{1}=19.5, j_{2}=20$ and the coupling strength $\epsilon=0.01$. The evolution time comprises 1000 kicks. The statistical limit (SL) for entanglement is drawn with the solid line and corresponds to the average entanglement of a randomly chosen state of the composite system. The initial entanglement growth is higher for regular dynamics (low $k$ ) than for the chaotic one (high $k$ ). While asymptotic values of entanglement are high for chaotic cases, the asymptotic value for the most regular dynamics $k=0.01$ outperforms all other cases.

values can be observed for $k=2,4,6$. However, the differences in the asymptotic values for $k=4,6$ are tiny. The $k=0.01$ case is especially interesting. During 1000 kicks the entanglement saturates to an extremely high value - higher than for all other values of $k$. Again, a nonmonotonic $k$-dependence of the asymptotic entanglement is observed.

Studying the initial production rate of entanglement one can observe anticorrelation with the parameter $k$. The fastest initial growth of entanglement corresponds to $k=0.01$, and the slowest to highly chaotic cases $k=4,6$ - chaos suppresses the initial entanglement production rate.

For shortening the notation we shall denote the $\mathrm{SU}(2) \times \mathrm{SU}(2)$ averaging by the $\mathrm{SU}(2)$ averaging and the $\mathrm{SU}\left(d_{1}\right) \times \mathrm{SU}\left(d_{2}\right)$ by the $\mathrm{SU}(d)$ averaging. The $\mathrm{SU}(d)$ averaged behavior of initial entanglement growth is strikingly different as compared with the $\mathrm{SU}(2)$ averaging. In the latter case the initial entanglement production rate was almost zero for low values of $k$, while in the former it was extremely high. Consequently, one should always distinguish between the entangling power of an evolution and its particular entangling properties in acting on a certain group of states as these two may behave very differently.

Summing up the qualitative discussion we conclude that the entangling power of the evolution [correspond- 


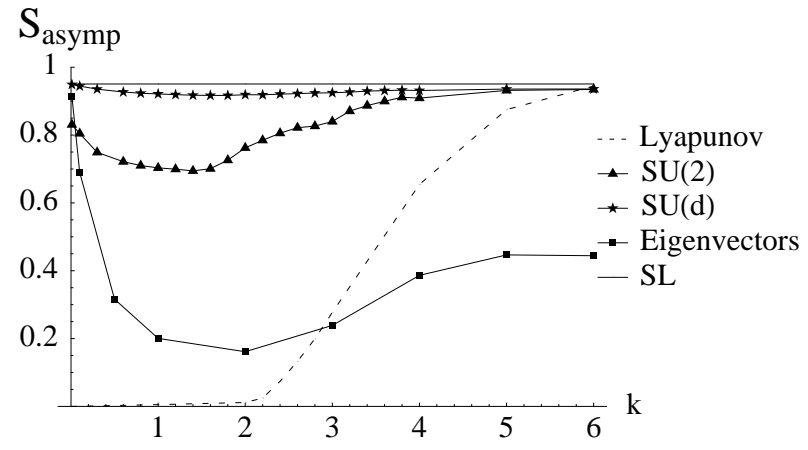

FIG. 3: This figure presents the dependence of asymptotic values of entanglement on the chaoticity parameter $k$. Asymptotic values of entanglement corresponding to averaging over initial random product states [the $\mathrm{SU}(d)$ averaging] are denoted by stars and asymptotic values corresponding to the averaging over initial spin-coherent product states [the $\mathrm{SU}(2)$ averaging] are denoted by triangles. The dashed line represents the Lyapunov exponent of the classical dynamics of a single top. The mean entanglement of eigenvectors of the evolution is denoted by rectangles. Statistical limit (SL) for entanglement is drawn with the solid line and corresponds to average entanglement of a randomly chosen state of the composite system. Chaos indeed increases asymptotic value of entanglement in the case of spin-coherent states and, to some extent, also in the case of random product states; nevertheless very regular regime $k \approx 0$ also manifests high asymptotic entanglement which can be related to high entanglement of eigenvectors.

ing to $\mathrm{SU}(d)$ averaging] both in terms of the asymptotic value and the initial growth is the highest for very low $k$ - i.e. for very regular dynamics.

\section{B. Asymptotic behavior}

We give here more detailed results on asymptotic entanglement. In Fig. 3 the asymptotic values of entanglement are presented for different values of $k$. For more credible results the asymptotic values were obtained as averages of the linear entropy over the evolution of the tops between 50,000 and 100,000 kicks. Stars correspond to $\mathrm{SU}(d)$ averaging while triangles correspond to $\mathrm{SU}(2)$ averaging. The Lyapunov exponent obtained from the classical dynamics of a single top is shown by the dashed line. For the sake of later discussion we also included the mean entanglements of eigenvectors of the evolution operator - denoted by rectangles.

In the case of $\mathrm{SU}(2)$ averaging, for very low values of $k$, the asymptotic values are high. With the increase of $k$ they decrease, reaching minimum for $k \approx 1.5$, subsequently, with the onset of chaos, they increase again and eventually saturate. The saturation value is a little below the statistical limit (SL).

The $\mathrm{SU}(d)$ averaging reveals almost no dependence of

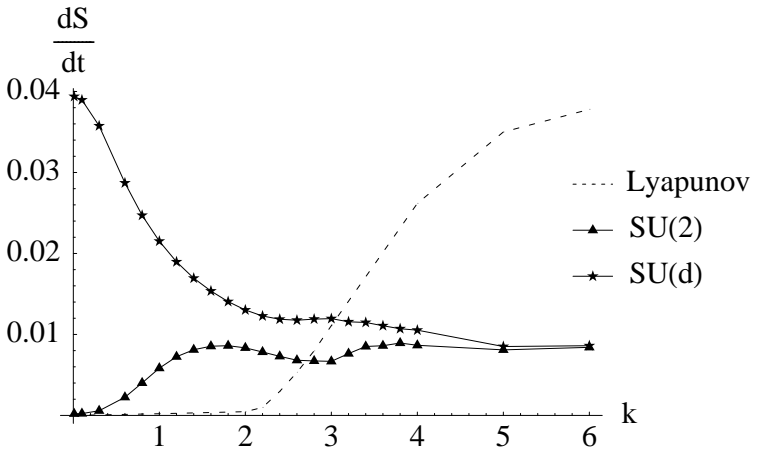

FIG. 4: Dependence of initial entanglement production rate on the chaoticity parameter $k$. Results obtained after averaging over initial random product states [the $\mathrm{SU}(d)$ averaging] are denoted by stars and results due to averaging over initial spin-coherent product states [the $\mathrm{SU}(2)$ averaging] are denoted by triangles. The dashed line represents the rescaled Lyapunov exponent of the classical dynamics of a single top. In the case of random product states the highest initial entanglement growth corresponds to very regular dynamics and diminishes with the increase of the chaoticity parameter $k$ while in the case of spin-coherent product states it is very regular dynamics that has the slowest initial entanglement growth.

the asymptotic value of entanglement on $k$. Nevertheless, there is also a tiny dip around $k \approx 2$ and the entanglement for very low $k$ is a little bit higher than that for the strongly chaotic regime.

The behavior of the mean eigenvector entanglement, also reveals a minimum around $k \approx 2$. Remarkably the entanglement of eigenvectors for very high $k$ is significantly smaller than asymptotic value of entanglement in this regime.

\section{Initial behavior}

In order to grasp quantitatively the initial behavior of entanglement, we fit a line to the points representing short-time entanglement produced for certain value of $k$. Although the character of initial entanglement growth is linear only in the chaotic regime, while in the regular regime it is quadratic (see Sec. VIC), we perform the fitting in all regimes. This gives us sensible estimate of initial entanglement growth. The fitting is done for points corresponding to first 15 kicks. Regression coefficients obtained in this way are shown in Fig. 4 for both $\mathrm{SU}(2)$ and $\mathrm{SU}(d)$ averaging, together with the rescaled Lyapunov exponent of a single top. For high values of $k$ both averaging methods give the same results, which is caused by strong chaos which even during the first 15 kicks is able to turn spin-coherent states into completely random states. For low values of $k$ the entanglement production rate obtained with the $\mathrm{SU}(d)$ averaging out- 
performs that of the $\mathrm{SU}(2)$ averaging. Furthermore it is approximately four times higher than in the strong chaos regime. We shall now move on to explaining these observations.

\section{THEORETICAL ANALYSIS OF NUMERICAL RESULTS}

Before moving on to separate discussions on the asymptotic and initial behavior of the entanglement production, let us first make a general remark which applies to both cases. Looking at Fig. 3 and Fig. 4 one can notice that when the chaoticity parameter is large $(k>5)$ differences between the $\mathrm{SU}(2)$ averaging and the $\mathrm{SU}(d)$ averaging disappear, both in results for the asymptotic and the initial behavior. It means that there is no difference, whether we choose as our initial states the ensemble of spin-coherent product states or the ensemble of random product states. Disappearance of the difference between two averaging methods for high $k$ is due to strong chaos which very quickly turns initial spin-coherent states into random pure states. The distinction between two averaging methods, however, is crucial in mixed and regular regimes.

\section{A. Entanglement of eigenvectors vs asymptotic values of entanglement of evolved states}

Abstracting for a while from the case of the kicked tops, let $U$ be a unitary operation acting in a Hilbert space $\mathcal{H}_{1} \otimes \mathcal{H}_{2}$, where dimensions of $\mathcal{H}_{1}, \mathcal{H}_{2}$ are respectively $d_{1}$ and $d_{2}$. We denote by $\left|e_{i}\right\rangle, \phi_{i}$ for $i=1 \ldots d_{1} d_{2}$ the eigenvectors and eigenphases of the operator $U$. We assume here that the spectrum is nondegenerate. The reduced density matrices of the eigenvectors after tracing out the second subsystem are $\rho_{i}=\operatorname{Tr}_{2}\left(\left|e_{i}\right\rangle\left\langle e_{i}\right|\right)$. As information about entanglement of an eigenvector we use again the linear entropy of its reduced density matrix: $S_{i}=1-\operatorname{Tr}\left(\rho_{i}^{2}\right)$. The mean entanglement of eigenvectors is given by

$$
\bar{S}_{\text {eigen }}=1-\frac{1}{d_{1} d_{2}} \sum_{i} \operatorname{Tr}\left(\rho_{i}^{2}\right) .
$$

Assume that the initial state of the system is $|\psi\rangle$. After $n$ iterations of the operation $U$ the resulting state reads

$$
|\psi(n)\rangle=\sum_{i} \exp \left(i n \phi_{i}\right)\left\langle e_{i} \mid \psi\right\rangle\left|e_{i}\right\rangle
$$

The reduced density matrix of the first subsystem corresponding to this state is given by

$$
\rho(n)=\sum_{i j} \exp \left[i n\left(\phi_{i}-\phi_{j}\right)\right]\left\langle e_{i} \mid \psi\right\rangle\left\langle\psi \mid e_{j}\right\rangle \operatorname{Tr}_{2}\left(\left|e_{i}\right\rangle\left\langle e_{j}\right|\right),
$$

the linear entropy of which reads

$$
\begin{aligned}
& S_{\rho(n)}=1-\operatorname{Tr}\left[\rho(n)^{2}\right]=1-\operatorname{Tr}\left(\sum_{i j k l} \exp \left[i n\left(\phi_{i}-\phi_{j}+\phi_{k}-\phi_{l}\right)\right]\right. \\
& \left.\left\langle e_{i} \mid \psi\right\rangle\left\langle\psi \mid e_{j}\right\rangle\left\langle e_{k} \mid \psi\right\rangle\left\langle\psi \mid e_{l}\right\rangle \operatorname{Tr}_{2}\left(\left|e_{i}\right\rangle\left\langle e_{j}\right|\right) \operatorname{Tr}_{2}\left(\left|e_{k}\right\rangle\left\langle e_{l}\right|\right)\right) .
\end{aligned}
$$

For the asymptotic behavior of the linear entropy the important terms in the above formula are those which survive after the time-averaging. These are the terms in which phase factors disappear. Other terms oscillate and thus vanish when averaged over time. The nonvanishing terms correspond to either $i=j, k=l$ or $i=l, k=j$. Asymptotic value of linear entropy reads thus

$$
\begin{aligned}
& S_{\text {asymp }}=1-\operatorname{Tr}\left(\sum_{i j}\left|\left\langle e_{i} \mid \psi\right\rangle\right|^{2}\left|\left\langle e_{j} \mid \psi\right\rangle\right|^{2} \rho_{i} \rho_{j}\right)- \\
& \operatorname{Tr}\left(\sum_{i \neq j}\left|\left\langle e_{i} \mid \psi\right\rangle\right|^{2}\left|\left\langle e_{j} \mid \psi\right\rangle\right|^{2} \operatorname{Tr}_{2}\left(\left|e_{i}\right\rangle\left\langle e_{j}\right|\right) \operatorname{Tr}_{2}\left(\left|e_{j}\right\rangle\left\langle e_{i}\right|\right)\right) .
\end{aligned}
$$

We would like to stress here that the above formula is the result of the averaging over time of the linear entropy itself. One could take a different approach and first average over time the density matrix and than calculate the linear entropy of the resulting density matrix. Following the second approach one would not get the third term in the above formula. This difference is important as it is certainly not the same to calculate the time-averaged entanglement of the evolved state or to calculate the entanglement of the time-averaged state. We argue that the first approach reveals better the entangling properties of the system evolution. One must admit, however, that in many cases the second approach will give qualitatively similar results.

We would like now to relate the above formula for the asymptotic entanglement of an evolved state with the entanglement of eigenvectors. We start by proving the following inequalities:

$$
\begin{aligned}
2 \operatorname{Tr}\left(\operatorname{Tr}_{2}\left(\left|e_{i}\right\rangle\left\langle e_{j}\right|\right) \operatorname{Tr}_{2}\left(\left|e_{j}\right\rangle\left\langle e_{i}\right|\right)\right) & \leq \operatorname{Tr}\left(\rho_{i}^{2}\right)+\operatorname{Tr}\left(\rho_{j}^{2}\right)(6.6 \mathrm{a}) \\
2 \operatorname{Tr}\left(\rho_{i} \rho_{j}\right) & \leq \operatorname{Tr}\left(\rho_{i}^{2}\right)+\operatorname{Tr}\left(\rho_{j}^{2}\right)(6.6 \mathrm{~b})
\end{aligned}
$$

Proof. We shall prove the first inequality. Let us decompose the eigenvector $\left|e_{i}\right\rangle$ in a product basis $\left|e_{i}\right\rangle=$ $\sum_{n_{1} n_{2}} C_{n_{1} n_{2}}^{i}\left|n_{1}\right\rangle \otimes\left|n_{2}\right\rangle$. We can write: $\operatorname{Tr}_{2}\left(\left|e_{i}\right\rangle\left\langle e_{j}\right|\right)=$ $C^{i} C^{j \dagger}$. It follows that

$$
\begin{array}{r}
2 \operatorname{Tr}\left[\operatorname{Tr}_{2}\left(\left|e_{i}\right\rangle\left\langle e_{j}\right|\right) \operatorname{Tr}_{2}\left(\left|e_{j}\right\rangle\left\langle e_{i}\right|\right)\right]= \\
=2 \operatorname{Tr}\left(C^{i} C^{j \dagger} C^{j} C^{i \dagger}\right)=2 \operatorname{Tr}\left(C^{i \dagger} C^{i} C^{j \dagger} C^{j}\right) \leq \\
\leq \operatorname{Tr}\left(C^{i \dagger} C^{i} C^{i \dagger} C^{i}\right)+\operatorname{Tr}\left(C^{j \dagger} C^{j} C^{j \dagger} C^{j}\right)=\operatorname{Tr}\left(\rho_{i}^{2}\right)+\operatorname{Tr}\left(\rho_{j}^{2}\right) .
\end{array}
$$

In the derivation above we have made use of the CauchySchwarz inequality. Assume that $A$ and $B$ are hermitian operators. The Cauchy-Schwarz inequality than reads $\operatorname{Tr}\left(A^{2}\right) \operatorname{Tr}\left(B^{2}\right) \geq[\operatorname{Tr}(A B)]^{2}$. Taking into account that $\left[\operatorname{Tr}\left(A^{2}\right)+\operatorname{Tr}\left(B^{2}\right)\right]^{2} \geq 4 \operatorname{Tr}\left(A^{2}\right) \operatorname{Tr}\left(B^{2}\right)$, one arrives at: 
$\operatorname{Tr}\left(A^{2}\right)+\operatorname{Tr}\left(B^{2}\right) \geq 2 \operatorname{Tr}(A B)$. This explains the derivation above and gives (6.6a). The proof of the inequality (6.6b) is analogous.

We now return to the formula (6.5). Using the inequalities (6.6) we can write:

$$
\begin{array}{r}
S_{\text {asymp }}=1-\operatorname{Tr}\left(\sum_{i j}\left|\left\langle e_{i} \mid \psi\right\rangle\right|^{2}\left|\left\langle e_{j} \mid \psi\right\rangle\right|^{2} \rho_{i} \rho_{j}\right)- \\
\operatorname{Tr}\left(\sum_{i \neq j}\left|\left\langle e_{i} \mid \psi\right\rangle\right|^{2}\left|\left\langle e_{j} \mid \psi\right\rangle\right|^{2} \operatorname{Tr}_{2}\left(\left|e_{i}\right\rangle\left\langle e_{j}\right|\right) \operatorname{Tr}_{2}\left(\left|e_{j}\right\rangle\left\langle e_{i}\right|\right)\right) \geq \\
\geq 1-\operatorname{Tr}\left(\sum_{i j}\left|\left\langle e_{i} \mid \psi\right\rangle\right|^{2}\left|\left\langle e_{j} \mid \psi\right\rangle\right|^{2} \rho_{i} \rho_{j}\right)- \\
\operatorname{Tr}\left(\sum_{i j}\left|\left\langle e_{i} \mid \psi\right\rangle\right|^{2}\left|\left\langle e_{j} \mid \psi\right\rangle\right|^{2} \operatorname{Tr}_{2}\left(\left|e_{i}\right\rangle\left\langle e_{j}\right|\right) \operatorname{Tr}_{2}\left(\left|e_{j}\right\rangle\left\langle e_{i}\right|\right)\right) \geq \\
\geq 1-2 \sum_{i}\left|\left\langle e_{i} \mid \psi\right\rangle\right|^{2} \operatorname{Tr}\left(\rho_{i}^{2}\right)=2 \sum_{i}\left|\left\langle e_{i} \mid \psi\right\rangle\right|^{2} S_{i}-1
\end{array}
$$

where $S_{i}$ is the linear entropy of the eigenstate $\left|e_{i}\right\rangle$.

We are interested in averaging over initial states $|\psi\rangle$ which are either spin-coherent product states or random product states. Both averaging procedures lead to

$$
\left\langle\left\langle\left|\left\langle e_{i} \mid \psi\right\rangle\right|^{2}\right\rangle\right\rangle_{\psi}=1 / d_{1} d_{2} .
$$

Finally we arrive at the following relation between mean asymptotic value of entanglement of evolved states and mean entanglement of eigenvectors:

$$
\bar{S}_{\text {asymp }} \geq 2 \bar{S}_{\text {eigen }}-1 .
$$

This inequality is valid for both the $\mathrm{SU}(2)$ averaging and the $\mathrm{SU}(d)$ averaging. Actually, as the equation (6.7) is valid for any ensemble of states which give a resolution of identity, so is the equation (6.8) valid in all these cases and not only in the case of $\mathrm{SU}(2)$ or $\mathrm{SU}(d)$ averaging. This inequality puts the lower bound on the mean asymptotic entanglement of evolved states, thus a high entanglement of eigenvectors induce a high mean asymptotic entanglement of evolved states.

One is not entitled, however, to claim that a low mean entanglement of eigenvectors implies a low mean asymptotic entanglement of evolved states (compare Fig. 3). To stress once again the inadequate information about entangling properties of a transformation obtained from studying its eigenvector entanglement, recall that even when all eigenvectors are product states, a transformation can have a non-zero entangling power (for example the controlled-phase gate).

This is a good place to comment on our decision to take $j_{1}$ different from $j_{2}$. If we took identical spins for the two tops the degeneracy of eigenvectors would appear. The presence of degeneracy invalidate the inequality (6.8). In the presence of degeneracy esti- mating entangling power from entanglement of eigenvectors can be even more misleading. As an example consider a local transformation $U=U_{1} \otimes U_{2}$, which obviously has the zero entangling power. Let $\left|f_{1}\right\rangle \otimes\left|f_{2}\right\rangle$ and $\left|g_{1}\right\rangle \otimes\left|g_{2}\right\rangle$ be two degenerate product eigenstates. However, eigenstates in this situation can equally well be taken as $(1 / \sqrt{2})\left(\left|f_{1}\right\rangle \otimes\left|f_{2}\right\rangle+\left|g_{1}\right\rangle \otimes\left|g_{2}\right\rangle\right)$ and $(1 / \sqrt{2})\left(\left|f_{1}\right\rangle \otimes\left|f_{2}\right\rangle-\left|g_{1}\right\rangle \otimes\left|g_{2}\right\rangle\right)$, which are entangled states. Consequently calculating numerically eigenstates and their entanglement we could arrive at the conclusion that the entanglement of eigenvectors is high while the transformation itself has the zero entangling power. Nondegenerate spectrum is thus an indispensable condition in studying relations between entangling power of a transformation and entanglement of its eigenvectors.

\section{B. Asymptotic behavior}

When explaining the asymptotic value of entanglement of an evolved state in a system of two strongly chaotic interacting subsystems one can formulate some statistical predictions. One expects that states evolved in such systems tend to generically random states. This means that writing an asymptotic state, in a product basis:

$$
\left|\psi_{\text {asymp }}\right\rangle=\sum_{n_{1} n_{2}} a_{n_{1} n_{2}}\left|n_{1}\right\rangle \otimes\left|n_{2}\right\rangle,
$$

one expects the coefficients $a_{n_{1} n_{2}}$ to be independent random variables. Depending on the symmetries of the system, one imposes different restrictions on the coefficients arriving thus at different state ensembles. It is then possible to calculate the linear entropy averaged over such ensembles. 18, 21]

Choosing an ensemble of states which are uniformly distributed with respect to the action of $\mathrm{SU}\left(d_{1} d_{2}\right)$ group, one arrives at averaged linear entropy [21]:

$$
S_{S L}=1-\frac{d_{1}+d_{2}}{d_{1} d_{2}+1} .
$$

Consequently, the above expression is equal to the average entanglement (measured as linear entropy of reduced density matrix) of a generically random state of the composite system. We shall use this expression, which we call statistical limit (SL), as a reference for the asymptotic entanglement of evolved states.

When dimensions of subspaces are similar $d_{1} \approx d_{2} \approx d$ and large the statistical limit can be written in a simple form $S_{S L} \approx 1-2 / d$. Compare this with the limit imposed solely by the dimensionality of the subspaces: $S^{\max }=$ $1-1 / d$, where $d$ is the dimension of the smaller subspace.

In Fig. 5 we compare this formula with the values of the asymptotic entanglement for strong chaos $k=6$, for different spin magnitudes of the two tops. There is no difference between values obtained for the $\mathrm{SU}(2)$ or the $\mathrm{SU}(d)$ averaging, and both are a little below the statistical limit (SL). Notice that the eigenvectors entanglement 


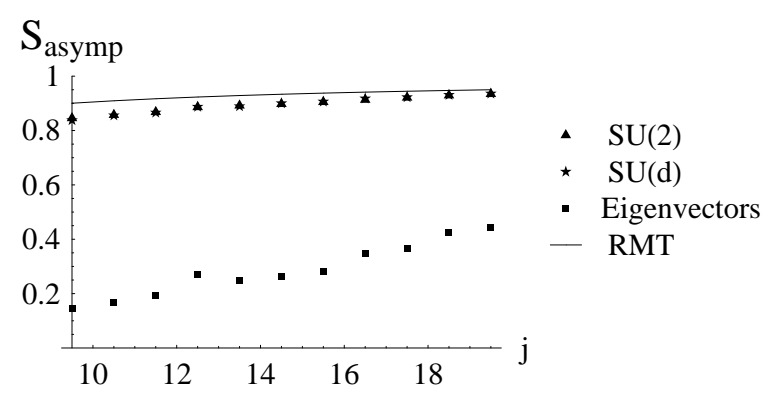

FIG. 5: Asymptotic entanglement of evolved states averaged over initial random product states [the $\mathrm{SU}(d)$ averaging, stars] and over initial spin-coherent product states [the SU(2) averaging, triangles) in the strong chaos regime $k=6$, calculated for different spin magnitudes $j$. Spins of the tops are chosen $j_{1}=j$ and $j_{2}=j+1 / 2$. Rectangles denote values of mean entanglement of eigenvectors. The statistical limit (SL) for entanglement is drawn with the solid line and corresponds to average entanglement of a randomly chosen state of the composite system. A big discrepancy is visible between the asymptotic entanglement of evolved states and the mean entanglement of eigenvectors.

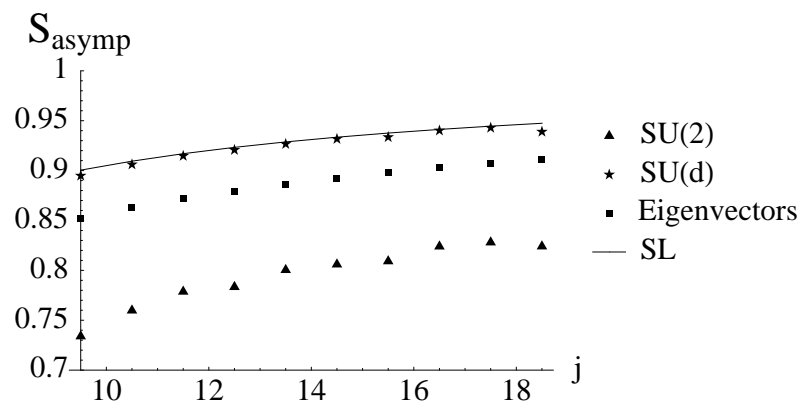

FIG. 6: Asymptotic entanglement of evolved states in the case of averaging over initial random product states $[\mathrm{SU}(d)$ averaging, stars] and in the case of averaging over initial spincoherent product states [SU(2) averaging, triangles] in the regular regime $k=0.01$, calculated for different spin magnitudes $j$. Spins of the tops are chosen: $j_{1}=j$ and $j_{2}=j+1 / 2$. Rectangles denote values of mean entanglement of eigenvectors. Statistical limit (SL) for entanglement is drawn with a solid line and corresponds to average entanglement of a randomly chosen state of the composite system. Thanks to inequality (6.8) high entanglement of eigenvectors is an indicator of high asymptotic entanglement for both averaging procedures

is hardly of any use here in predictions of the asymptotic entanglement.

We noticed earlier that for low values of $k$ asymptotic values of entanglement for the $\mathrm{SU}(2)$ and the $\mathrm{SU}(d)$ averaging are also very high. In the light of the inequality (6.8) this is due to the very high entanglement of the eigenvectors of the evolution operator. Asymptotic entanglements for $k=0.01$ are presented in Fig. [6 together with the entanglement of eigenvectors for different spin magnitudes. The statistical limit (SL) (6.10) is also plotted for comparison.

The agreement between the statistical limit (SL) and the asymptotic values of entanglement of the $\mathrm{SU}(d)$ averaging is purely accidental. This underlines, however, that the regular regime can be as good (or better) in generating high asymptotic values of entanglement for random states compared as the strongly chaotic regime. This agrees with the results in [23], where different approach was taken - no specific model of dynamics was analyzed but instead the Hamiltonians of the subsystems where random matrices chosen either from chaotic or regular ensembles.

It remains to be explained what is the reason for a very high entanglement of eigenvectors for low values of $k$. This effect of high entanglement of eigenvectors in the regular motion regime was also observed in the spinkicked rotor system [24] and a mechanism is similar here. We shall give a qualitative explanation of this effect. For decoupled kicked tops $\epsilon=0$, eigenvectors are product states. For low values of $k$ there are many states that are nearly degenerate. Additionally, these product states are very close to the product states of eigenvectors of the $J_{y}$ operator. Indeed for $k=0$ one time step of the evolution of the tops is described by the unitary operator:

$$
U_{0}=\exp \left(-i p J_{y_{1}}-i p J_{y_{2}}\right),
$$

so eigenvectors can be chosen as $\left|y_{1}, y_{2}\right\rangle$, the product states of the eigenvectors of $J_{y}$ operator. There are many degenerate eigenvectors of $U_{0}$, for example $|-j, j\rangle,|-j+1, j-1\rangle, \ldots,|j,-j\rangle-$ these are $2 j+1$ states with the eigenphase 0 (for simplicity we have assumed $j_{1}=j_{2}$ and the value of the spin to be integer). Additionally there will be also groups consisting of $2 j, 2 j-1, \ldots, 1$ degenerate states. The coupling term $\exp \left(-\epsilon J_{z 1} J_{z 2}\right)$, expanded to the first order in $\epsilon$, will couple the state $\left|y_{1}, y_{2}\right\rangle$ with the states of the form $\left|y_{1}-1, y_{2}+1\right\rangle,\left|y_{1}+1, y_{2}-1\right\rangle,\left|y_{1}+1, y_{2}+1\right\rangle,\left|y_{1}-1, y_{2}-1\right\rangle$ (as $J_{z}$ is a sum of lowering and rising operators in the basis of $J_{y}$ eigenvectors). A weak coupling between nondegenerate states will not cause much change in the form of eigenvectors. Let $\left|v_{i}\right\rangle$ be the set of degenerate eigenvectors of $U_{0}$. Due to the coupling, new eigenvectors will be obtained by diagonalizing a matrix of the approximately following form:

$$
\left[\begin{array}{cccc}
p & \epsilon & 0 & \cdots \\
\epsilon & p & \epsilon & \cdots \\
0 & \epsilon & p & \cdots \\
\vdots & \vdots & \vdots & \ddots
\end{array}\right] .
$$

Eigenvectors of such a matrix have large contributions from many different vectors $v_{i}$, in our case it means that in every subspace of degenerate product eigenvectors new highly entangled eigenvectors will emerge due to the weak coupling. 


\section{Initial behavior}

The initial entanglement growth in the system of coupled kicked tops can be well understood with the help of the perturbation theory developed in [7, 10].

It should be clarified here that, although the two papers [7, 10] contains similar results, the motivation and the scope of their work is a bit different. The main motivation of the work by Fujisaki et al. [7] is to study entanglement production in weakly coupled chaotic systems and as a model they consider the coupled kicked tops. This is also the approach we take in this article. On the other hand, Žnidarič and Prosen consider a problem of stability of quantum dynamics of a composite system with respect to weak interaction between two subsystems. They consider different quantities reflecting the stability of quantum motion such as fidelity, reduced fidelity and purity fidelity, and analyze their behavior under regular or chaotic dynamics for different times of evolution (comprehensive study of the problem of stability of quantum dynamics can be found in [25]), see also [8, 9, 26]). Because the linear entropy $(S)$, which is used as a measure of entanglement in [7] and in the present work, is related to the purity fidelity $\left(F_{P}\right)$ by the formula $S=1-F_{P}$, there is a close relation between results obtained in 7] and [10].

The essential quantity for understanding initial entanglement growth is the product of the time correlation functions of uncoupled subsystems:

$$
D(n, m)=C_{1}(n, m) C_{2}(n, m),
$$

where the correlation function of an uncoupled subsystem is defined

$$
C_{i}(n, m)=\left\langle\hat{z}_{i}(n) \hat{z}_{i}(m)\right\rangle-\left\langle\hat{z}_{i}(n)\right\rangle\left\langle\hat{z}_{i}(m)\right.
$$

and $\hat{z}_{i}(m)=J_{z_{i}}(m) / j_{i}$. The Heisenberg picture is used here and $J_{z_{i}}(n)$ denotes the operator of projection of the angular momentum on the $z$ axis at time $n$ for the subsystem $i$ (after the $n$-th kick of uncoupled evolution).

The perturbation formula for the initial behavior of the linear entropy reads

$$
S^{\text {perturb }}(t)=2 \epsilon^{2} j_{1} j_{2} \sum_{n=1}^{t} \sum_{m=1}^{t} D(n, m) .
$$

For strong chaos one can neglect the terms with $n \neq m$, as any correlation is quickly washed out. For $n=m$ due to the chaotic character of the motion $D(n, n) \approx 1 / 9$ and does not depend on $n$ [7] (more detailed analysis of the behavior of the correlation function can be found in $[6,7,[8,[10])$. Finally for strong chaos one obtains that the initial growth of linear entropy is linear, and the rate of the growth is given by:

$$
\frac{d S^{\text {perturb }}}{d t}=\frac{2}{9} \epsilon^{2} j_{1} j_{2}
$$

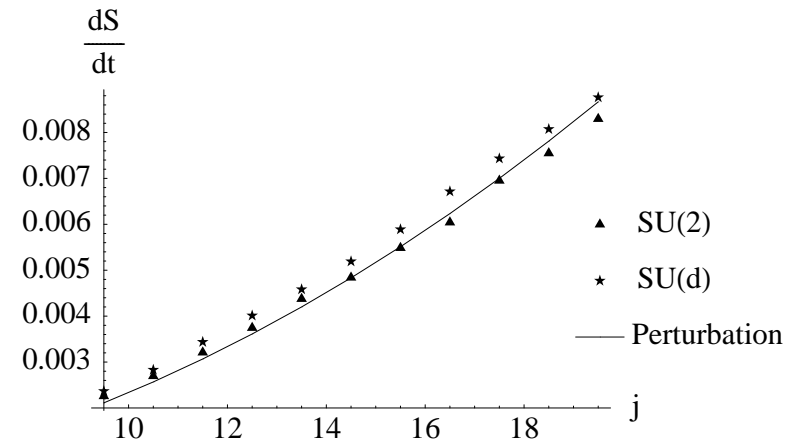

FIG. 7: Initial entanglement growth rates averaged over initial random product states [the $\mathrm{SU}(d)$ averaging - stars] and initial entanglement growth rates averaged over initial spincoherent product states [the $\mathrm{SU}(2)$ averaging - triangles]. The results are obtained in strongly chaotic regime $k=6$ and for different spin magnitudes $j$. The spins of the tops differ by $1 / 2: \quad j_{1}=j$ and $j 2=j+1 / 2$. The prediction of perturbative formula (6.15) is drawn with the solid line. Due to strong chaos there is no significant difference between the spin-coherent and the random states.

This formula is valid for any initial state, actually not from the very beginning of the evolution, but only after a short relaxation time when the linear growth of the linear entropy emerges. In our case the linear growth appears very quickly, after first few kicks.

The comparison of the above formula with the initial entanglement growth rate corresponding to the $\mathrm{SU}(2)$ and the $\mathrm{SU}(d)$ averaging, in the case of strong chaos $k=6$ is shown in Fig. 7. The agreement is good and, due to strong chaos, there is no significant difference between two ensembles.

In the regular regime, the formula (6.14), yields initial quadratic increase of linear entropy, due to nonvanishing correlations $C(n, m)$ for $n \neq m$ [7, 9]. In this regime there is qualitatively different behavior of linear entropy increase between $\mathrm{SU}(2)$ and $\mathrm{SU}(d)$ averaging (see Fig. (4).

In order to explain the difference we have to estimate values of the correlation functions $C(n, m)$ averaged over the $\mathrm{SU}(2)$ and $\mathrm{SU}(d)$ ensembles of initial states. Notice the following formulas for a particle with spin $j$ :

$$
\begin{aligned}
\int d \mu_{\mathrm{SU}(2)}\left\langle\psi\left|J_{z}^{2}\right| \psi\right\rangle & =\frac{j(j+1)}{3}, \\
\int d \mu_{\mathrm{SU}(2)}\left\langle\psi\left|J_{z}\right| \psi\right\rangle^{2} & =\frac{j^{2}}{3}, \\
\int d \mu_{\mathrm{SU}(d)}\left\langle\psi\left|J_{z}^{2}\right| \psi\right\rangle & =\frac{j(j+1)}{3}, \\
\int d \mu_{\mathrm{SU}(d)}\left\langle\psi\left|J_{z}\right| \psi\right\rangle^{2} & =\frac{j}{6},
\end{aligned}
$$

where $d=2 j+1$ is the dimension of the particle's Hilbert space. Proofs of these formulas are given in the appendix. The same equations can be written when $J_{z}$ is replaced 
by either $J_{y}$ or $J_{x}$. If, however, $\left\langle\psi\left|J_{z}^{2}\right| \psi\right\rangle$ is replaced by a mixed term - for example $\left\langle\psi\left|J_{z} J_{x}\right| \psi\right\rangle$ - the integrals 6.16a , 6.16c vanish. The same happens if in the integrals (6.16b), 6.16b the term $\left\langle\psi\left|J_{z}\right| \psi\right\rangle^{2}$ is replaced by a mixed term - for example $\left\langle\psi\left|J_{z}\right| \psi\right\rangle\left\langle\psi\left|J_{x}\right| \psi\right\rangle$.

In the most regular case $(k=0.01)$ the evolution of a single top is mostly determined by its free rotation around $y$ axis. Each period corresponds to a $\pi / 2$ rotation. Consequently in the Heisenberg picture operator $J_{z}$ evolves approximately in the following way: $J_{z}(1) \approx J_{x}$, $J_{z}(2) \approx-J_{z}, J_{z}(3) \approx-J_{x}, J_{z}(4) \approx J_{z} \ldots$. The value of the correlation at equal times $C(n, n)$ is just the dispersion of $J_{z}(n)$ operator. In the case of the $\mathrm{SU}(2)$ averaging this will be proportional to the difference of integrals (6.16a and (6.16b) and thus proportional to $1 / j$. In contrast, $C(n, n)$ for the $\mathrm{SU}(d)$ averaging is proportional to the difference of integrals (6.16c) and 6.16d and thus proportional to 1 - the same order of magnitude as in the chaotic case. The same fact will hold for correlation functions calculated for times differing by even number of kicks, whereas correlation functions calculated for times differing by odd number of kicks will be zero. As $D(n, m)$ is a product of two correlation functions for the two tops, it will be approximately $j_{1} j_{2}$ times greater for the $\mathrm{SU}(d)$ averaging than for the $\mathrm{SU}(2)$ averaging. In the case we consider, this amounts to the ratio of initial entanglement production rate of approximately 400. This explains the strikingly different behavior of the initial entanglement growth for two considered kinds of averaging in for the $k=0.01$ case. Different character of entanglement production rate for coherent and random states was also noticed in [9].

$\mathrm{SU}(d)$ averaging reveals decrease in the initial entanglement growth rate with the increase of chaoticity parameter $k$ As the states we average over the ensemble of random states invariant under the action of $\mathrm{SU}(d)$, the increase of parameter $k$ does not change the value of the dispersion of $J_{z}$ operator; thus $C(n, n)$ does not depend on $k$. At the same time the increase of $k$ decreases correlation for different times $C(n, m)(n \neq m)$, and consequently the increase of the chaoticity parameter checks initial entanglement growth.

In the case of $\mathrm{SU}(2)$ averaging, consequences of the increase of the chaoticity parameter are twofold. On the one hand the increase of chaos decreases correlations $C(n, m)$ for $n \neq m$, and in this way transforms initial entanglement growth character from quadratic to linear. On the other hand it drives well-localized spin-coherent states into more smeared states with larger dispersion of $J_{z}$ operator, which results in the increase of $C(n, n)$ function calculated at equal times. Depending on the specific values of parameters of the system: $j_{1}, j_{2}, \epsilon$, one of theses factors may bare grater significance, and it may happen, that the increase of chaos corresponds to either the increase or decrease of initial entanglement growth (see for example numerical results in [12] for different values of $\epsilon$ parameter). We chose the parameters that reveal the increase of entanglement growth with growing $k$ for $\mathrm{SU}(2)$ averaging in order to contrast this with the behavior of entanglement growth in the case of $\mathrm{SU}(d)$ averaging, as for the latter case the increase of chaos always diminishes initial entanglement growth.

\section{CONCLUSIONS}

In this paper, the problem of the interplay between entanglement production in a quantum system and its chaotic properties was analyzed using the model of the coupled kicked tops.

Entangling properties of the coupled kicked tops system were investigated by observing the evolution of two different ensembles of product states. Considerations of ensembles consisting either of product spincoherent states [SU(2) averaging] or random product states $[\mathrm{SU}(d]$ averaging) lead to qualitatively different results in terms of the initial entanglement production rate and the asymptotic entanglement of evolved states. The asymptotic values of entanglement are high in a strongly chaotic regime (high kick strength), still they are even higher for a very regular regime (low kick strength), and reach minimum for the parameters of the evolution that correspond to the onset of chaos.

The SU(2) averaging reveals strong dependence of the asymptotic value of entanglement on the kick strength, while asymptotic values obtained after the $\mathrm{SU}(d)$ averaging are quite insensitive to variation of this parameter. This is due to more classical nature of spin-coherent states, which "feel" more directly the transition form regular to chaotic dynamics in the classical motion. In the case of the $\mathrm{SU}(d)$ averaging, the classical transition from regular to chaotic motion has only minor influence on the asymptotic value of entanglement.

In order to gain a deeper understanding of the problems, the asymptotic entanglement behavior was related to the mean entanglement of eigenvectors of the transformation. We have proved an inequality relating these two quantities. We have also pointed out cases where this quantities differ strongly and one is not entitled to infer anything about one of them from the value of the other.

Finally we have studied the averaged initial entanglement production rate. The striking difference between the $\mathrm{SU}(2)$ and the $\mathrm{SU}(d)$ averaging was explained with the help of the perturbation theory [7, 10]. In the regular regime spin-coherent product states become entangled much slower than random product states, due to a significantly smaller value of averaged variances of angular momentum components.

Regular to chaotic transition can indeed be observed in the global entangling properties of the kicked tops. These manifestations, however, are different depending on the ensembles of states considered. When considering random product states the increase of the chaoticity parameter always diminishes initial entanglement growth. For spin-coherent states increase of chaos results in two competing tendencies: chaos drives coherent states into more 
delocalized states and thus helps entanglement growth, but on the other hand it destroys time correlations in subsystems, which checks entanglement growth. We chose parameters of the evolution in which first tendency prevailed and thus contrasted the behavior of spin-coherent vs. random states.

When discussing asymptotic entanglement we have observed that both purely regular and strongly chaotic regimes enjoy a very high asymptotic entanglement. Reasons for high asymptotic entanglement in regular and chaotic cases are different. In the chaotic case, chaotic dynamics in subsystems allows for a coupling of arbitrary states and consequently an initial product state can become highly entangled as the dynamics is able to penetrate Hilbert space of the composite system with almost no constraints. In the regular case evolution of a state is much more restricted, but in the system of coupled kicked tops, due to a very high entanglement of eigenvectors (see inequality 6.8) the asymptotic entanglement is also very high.

\section{Acknowledgments}

We would like to thank T. Prosen and M. Žnidarič for informing us about references [8, 9, 10]. This work was supported by the EC grant QUPRODIS, contract No IST-2001-38877 and the Polish Ministry of Scientific Research and Information Technology under the (solicited) grant No PBZ-Min-008/P03/03.

\section{APPENDIX: DERIVATION OF FORMULAS (6.16)}

Formulas (6.16a, 6.16c) can be explained using symmetry arguments. If instead of $J_{z}^{2}$, we took $J^{2}=$ $J_{x}^{2}+J_{y}^{2}+J_{z}^{2}$, the mean value $\left\langle\psi\left|J^{2}\right| \psi\right\rangle=j(j+1)$, for any state. Both $\mathrm{SU}(2)$ and $\mathrm{SU}(d)$ averaging do not distinguish any direction in space. Consequently averages of $J_{x}^{2}, J_{y}^{2}$ and $J_{z}^{2}$ should be equal. In order to sum up to $j(j+1)$ for $J^{2}$ operator each of these averages must be equal $j(j+1) / 3$

Let us now prove formula 6.16b). A spin-coherent state can be obtained as a rotation of $|j\rangle$ state: $|\theta, \phi\rangle=$ $R(\theta, \phi)|j\rangle$. This allows us to write

$$
\begin{array}{r}
\int d \mu_{\mathrm{SU}(2)}\left\langle\psi\left|J_{z}\right| \psi\right\rangle^{2}=\frac{1}{4 \pi} \int d \theta d \phi \sin (\theta)\left\langle\theta, \phi\left|J_{z}\right| \theta, \phi\right\rangle^{2}= \\
=\frac{1}{4 \pi} \int d \theta d \phi \sin (\theta)\left\langle-j\left|R(\theta, \phi)^{\dagger} J_{z} R(\theta, \phi)\right|-j\right\rangle^{2}= \\
=\frac{1}{4 \pi} \int d \theta d \phi \sin (\theta)\langle-j| J_{z} \cos (\theta)+J_{x} \sin (\theta) \cos (\phi) \\
-J_{y} \sin (\theta) \sin (\phi)|-j\rangle^{2}=\frac{1}{4 \pi} \int d \theta d \phi \sin (\theta) \cos ^{2}(\theta) j^{2}=\frac{j^{2}}{3} .
\end{array}
$$

$\sum_{m=-j}^{j} a_{m}|m\rangle$, where $a_{m}=x_{m}+i y_{m}$ are complex coefficients. The $\mathrm{SU}(d)$ averaging over $|\psi\rangle$ results in a random distribution of $a_{m}$ coefficients with the probability distribution:

$$
\begin{array}{r}
P\left(a_{-j}, \ldots, a_{j}\right)=P\left(x_{-j}, y_{-j}, \ldots, x_{j}, y_{j}\right)= \\
=\frac{(2 j) !}{\pi^{2 j+1}} \delta\left(1-\sum_{m=-j}^{j} x_{m}^{2}-\sum_{m=-j}^{j} y_{m}^{2}\right) .
\end{array}
$$

Marginal distributions can be calculated from the above formula. In what follows we shall need the two lowest marginal distributions of $P$ i.e. $P_{1}(x)$ and $P_{2}(x, y)$ [27]:

$$
\begin{array}{r}
P_{1}(x)=\frac{1}{\sqrt{\pi}} \frac{\Gamma(2 j+1)}{\Gamma(2 j+1 / 2)}\left(1-x^{2}\right)^{2 j-\frac{1}{2}} \\
P_{2}(x, y)=\frac{(2 j) !}{\pi}\left(1-x^{2}-y^{2}\right)^{2 j-1} .
\end{array}
$$

Note also the following equalities:

$$
\begin{aligned}
\int d x x^{4} P_{1}(x) & =\frac{3}{8(j+1)(2 j+1)} \\
\int d x d y x^{2} y^{2} P_{2}(x, y) & =\frac{1}{8(j+1)(2 j+1)}
\end{aligned}
$$

We are now prepared to prove formula (6.16d):

$$
\begin{array}{r}
\int d \mu_{\mathrm{SU}(d)}\left\langle\psi\left|J_{z}\right| \psi\right\rangle^{2}=\int d a_{-j} \ldots d a_{j} P\left(a_{-j}, \ldots a_{j}\right) \\
\left(\sum_{n=-j}^{j} a_{n}^{*}\left\langle n\left|J_{z} \sum_{m=-j}^{j} a_{m}\right| m\right\rangle\right)^{2}= \\
\int d a_{-j} \ldots d a_{j} P\left(a_{-j}, \ldots a_{j}\right)\left(\sum_{m=-j}^{j}\left|a_{m}\right|^{2} m\right)^{2}= \\
\int d a_{-j} \ldots d a_{j} P\left(a_{-j}, \ldots a_{j}\right) \sum_{m=-j}^{j} \sum_{n=-j}^{j}\left|a_{m}\right|^{2}\left|a_{n}\right|^{2} m n
\end{array}
$$

The proof of formula (6.16d) requires a little more effort. An arbitrary state $|\psi\rangle$ can be written as: $|\psi\rangle=$ only the terms with either $n=m$ or $n=-m$ contribute, 
as all others cancel out:

$$
\begin{array}{r}
\int d a_{-j} \ldots d a_{j} P\left(a_{-j}, \ldots a_{j}\right) \sum_{m=-j}^{j}\left|a_{m}\right|^{4} m^{2}- \\
\sum_{m=-j}^{j}\left|a_{m}\right|^{2}\left|a_{-m}\right|^{2} m^{2}= \\
\int d x_{-j} d y_{-j} \ldots d x_{j} d y_{j} P\left(x_{-j}, y_{-j}, \ldots, x_{j}, y_{j}\right) \\
\sum_{m=-j}^{j}\left(x_{m}^{4}+y_{m}^{4}+2 x_{m}^{2} y_{m}^{2}\right) m^{2}- \\
\sum_{m=-j}^{j}\left(x_{m}^{2}+y_{m}^{2}\right)\left(x_{-m}^{2}+y_{-m}^{2}\right) m^{2}= \\
\left(2 \int d x x^{4} P_{1}(x)-2 \int d x d y x^{2} y^{2} P_{2}(x, y)\right) \sum_{m=-j}^{j} m^{2}= \\
\frac{1}{2(j+1)(2 j+1)} \frac{j(j+1)(2 j+1)}{3}=\frac{j}{6} .
\end{array}
$$

[1] F. Haake, Quantum signatures of chaos (Springer-Verlag, 1991).

[2] L. E. Reichl, The transition to chaos (Springer-Verlag, 1992).

[3] K. Furuya, M. C. Nemes, and G. Q. Pellegrino, Phys. Rev. Lett. 80, 5524 (1997).

[4] P. A. Miller and S. Sarkar, Phys. Rev. E 60, 1542 (1998).

[5] R. M. Angelo, K. Furuya, M. C. Nemes, and G. Q. Pellegrino, Phys. Rev. E 60, 5407 (1999).

[6] A.Tanaka, H. Fujisaki, and T. Miyadera, Phys. Rev. E 66, 045201(R) (2002).

[7] H. Fujisaki, T. Miyadera, and A. Tanaka, Phys. Rev. E 67, 066201 (2003).

[8] T. Prosen and T. H. Seligman, J. Phys. A: Math. Gen. 35, 4707 (2002).

[9] T. Prosen, T. H. Seligman, and M. Žnidarič, Phys. Rev. A 67, $042112(2003)$.

[10] M. Žnidarič and T. Prosen, J. Phys. A: Math. Gen. 36, 2463 (2003).

[11] X. Wang, S. Ghose, B. Sanders, and B. Hu, quant$\mathrm{ph} / 0312047$ (2003).

[12] J. N. Bandyopadhyay and A. Lakshminarayan, Phys. Rev. E 69, 016201 (2004).

[13] A. Lakshminarayan, Phys. Rev. E 64, 036207 (2001).

[14] P. Zanardi, C. Zalka, and L. Faoro, Phys. Rev. A 62,
030301(R) (2000)

[15] R. F. Werner, Phys. Rev. A 40, 4277 (1989).

[16] C. H. Bennett, H. J. Bernstein, S. Popescu, and B. Schumacher, Phys. Rev. A 53, 2046 (1996).

[17] G. Vidal, J. Mod. Opt 47, 355 (2000).

[18] J. N. Bandyopadhyay and A. Lakshminarayan, Phys. Rev. Lett. 89, 060402 (2002).

[19] M. A. Nielsen and I. L. Chuang, Quantum Computing and Quantum Information (Cambridge University Press, 2000).

[20] F. Haake, M. Kuś, and R. Scharf, Z. Phys. B 65, 381 (1987).

[21] K. Życzkowski and H. J. Sommers, J. Phys. A: Math. Gen. 34, 7111 (2001).

[22] M. Poźniak, K. Życzkowski, and M. Kuś, J. Phys. A: Math. Gen. 31, 1059 (1998).

[23] T. Gorin and T. H. Seligman, J. Opt. B: Quantum Semiclass. Opt. 4, S386 (2002), URL quant-ph/0112030.

[24] A. Tanaka, J. Phys. A: Math. Gen. 29, 5475 (1996).

[25] M. Žnidarič, quant-ph/0406123 (2004).

[26] T. Prosen, T. H. Seligman, and M. Žnidarič, Prog. Theo. Phys. Supp. 150, 200 (2003), URL quant-ph/0304104.

[27] M. Kuś, J. Mostowski, and F. Haake, J. Phys. A: Math. Gen. 21, L1073 (1988). 